\title{
F-STABLE SECONDARY REPRESENTATIONS AND DEFORMATION OF F-INJECTIVITY
}

\author{
ALESSANDRO DE STEFANI AND LINQUAN MA
}

\begin{abstract}
We prove that deformation of F-injectivity holds for local rings $(R, \mathfrak{m})$ that admit secondary representations of $H_{\mathfrak{m}}^{i}(R)$ which are stable under the natural Frobenius action. As a consequence, F-injectivity deforms when $(R, \mathfrak{m})$ is sequentially Cohen-Macaulay (or more generally when all the local cohomology modules $H_{\mathfrak{m}}^{i}(R)$ have no embedded attached primes). We obtain some additional cases if $R / \mathfrak{m}$ is perfect or if $R$ is $\mathbb{N}$-graded.
\end{abstract}

\section{INTRODUCTION}

Throughout this article, all rings are commutative, Noetherian, and with multiplicative identity. For rings containing a field of characteristic $p>0$, the seminal work of Hochster and Huneke on tight closure, and subsequent works of many others, has led to a systematic study of the so-called F-singularities. Roughly speaking, these are singularities that can be defined using the Frobenius endomorphism $F: R \rightarrow R$, which is the map that raises every element of $R$ to its $p$-th power. One of the most studied F-singularities is F-injectivity, which is defined in terms of injectivity of the natural Frobenius actions on the local cohomology modules $H_{\mathfrak{m}}^{i}(R)$. It was first introduced and studied by Fedder in [Fed83].

We say that a property $\mathcal{P}$ of local rings deforms if, whenever $(R, \mathfrak{m})$ is a local ring and $x \in \mathfrak{m}$ is a nonzerodivisor such that $R /(x)$ satisfies $\mathcal{P}$, then $R$ satisfies $\mathcal{P}$. While this deformation problem for other classical F-singularities has been settled [Fed83, HH94, Sin99b, Sin99a], whether F-injectivity deforms or not in general is still an open question. Fedder proved that F-injectivity deforms when $R$ is Cohen-Macaulay [Fed83, Theorem 3.4], and Horiuchi, Miller, Shimomoto proved that F-injectivity deforms either if $R /(x)$ is F-split [HMS14, Theorem 4.13], or if $H_{\mathfrak{m}}^{i}(R /(x))$ has finite length for all $i \neq \operatorname{dim}(R)$ and $R / \mathfrak{m}$ is perfect [HMS14, Theorem 4.7]. More recently, the second author and Pham [MQ18] extended some of these results by further relaxing the assumptions on $R /(x)$.

In this paper, we consider secondary representations of the local cohomology modules $H_{\mathfrak{m}}^{i}(R)$ (see subsection 2.2 for definitions and basic properties of secondary representations of Artinian modules). It seems natural to ask how the Frobenius action on $H_{\mathfrak{m}}^{i}(R)$ interacts with a given secondary representation. Our first main result is that F-injectivity deforms when each local cohomology module $H_{\mathfrak{m}}^{i}(R)$ admits a secondary representation which is stable under the natural Frobenius action (see Definition 3.1 for details).

Theorem A (Theorem 3.4). Let $(R, \mathfrak{m})$ be a d-dimensional local ring of characteristic $p>0$ and let $x \in \mathfrak{m}$ be a nonzerodivisor on $R$. Suppose for each $i \neq d, H_{\mathfrak{m}}^{i}(R)$ has an F-stable secondary representation. If $R /(x)$ is F-injective, then $R$ is F-injective.

The second author was supported by NSF Grant DMS \#1901672, NSF FRG Grant DMS \#1952366, and a fellowship from the Sloan Foundation. 
We prove that secondary components that correspond to minimal attached primes of $H_{\mathfrak{m}}^{i}(R)$ are always F-stable, see Lemma 3.2. As a consequence, F-injectivity deforms when the attached primes of $H_{\mathfrak{m}}^{i}(R)$ are all minimal, see Corollary 3.5 for a slightly stronger statement. In particular, we obtain the following:

Corollary B (Corollary 3.6). Let $(R, \mathfrak{m})$ be a d-dimensional sequentially Cohen-Macaulay local ring of characteristic $p>0$ and let $x \in \mathfrak{m}$ be a nonzerodivisor on $R$. If $R /(x)$ is F-injective, then $R$ is F-injective.

We can further relax our assumptions if either the residue field of $R$ is perfect, or if $R$ is $\mathbb{N}$-graded over a field, by only putting conditions on those secondary components of $H_{\mathfrak{m}}^{i}(R)$ whose attached primes are not equal to $\mathfrak{m}$. We refer to Definition 3.7 for the precise meaning of $\mathrm{F}^{\circ}$-stable secondary representations.

Theorem $\mathbf{C}$ (Theorem 3.8 and Theorem 3.10). Let $(R, \mathfrak{m}, k)$ be a d-dimensional local ring of characteristic $p>0$ that is either local with perfect residue field or $\mathbb{N}$-graded over a field $k$, and let $x \in \mathfrak{m}$ be a nonzerodivisor on $R$ (homogeneous in the graded case). Suppose for each $i \neq d, H_{\mathfrak{m}}^{i}(R)$ has an $\mathrm{F}^{\circ}$-stable secondary representation. If $R /(x)$ is F-injective, then $R$ is F-injective.

Acknowledgments. We thank Pham Hung Quy and Ilya Smirnov for several useful discussions on the topics of this article.

\section{Preliminaries}

2.1. Frobenius actions on local cohomology and F-injectivity. Let $R$ be a ring of characteristic $p>0$. A Frobenius action on an $R$-module $W$ is an additive map $F: W \rightarrow W$ such that $F(r \eta)=r^{p} F(\eta)$ for all $r \in R$ and $\eta \in W$.

Let $I=\left(f_{1}, \ldots, f_{n}\right)$ be an ideal of $R$, then we have the Čech complex:

$$
C^{\bullet}\left(f_{1}, \ldots, f_{n} ; R\right):=0 \rightarrow R \rightarrow \oplus_{i} R_{f_{i}} \rightarrow \cdots \rightarrow R_{f_{1} f_{2} \cdots f_{n}} \rightarrow 0 .
$$

Since the Frobenius endomorphism on $R$ induces the Frobenius endomorphism on all localizations of $R$, it induces a natural Frobenius action on $C^{\bullet}\left(f_{1}, \ldots, f_{n} ; R\right)$, and hence it induces a natural Frobenius action on each $H_{I}^{i}(R)$. In particular, there is a natural Frobenius action $F: H_{\mathfrak{m}}^{i}(R) \rightarrow H_{\mathfrak{m}}^{i}(R)$ on each local cohomology module of $R$ supported at a maximal ideal $\mathfrak{m}$. A local ring $(R, \mathfrak{m})$ is called F-injective if $F: H_{\mathfrak{m}}^{i}(R) \rightarrow H_{\mathfrak{m}}^{i}(R)$ is injective for all $i$.

2.2. Secondary representations. We recall some well-known facts on secondary representations that we will use throughout this article. For unexplained facts, or further details, we refer the reader to [BS13, Section 7.2].

Definition 2.1. Let $R$ be a ring. An $R$-module $W$ is called secondary if $W \neq 0$ and for each $x \in R$ the multiplication by $x$ map on $W$ is either surjective or nilpotent.

One can easily check that, if $W$ is a secondary $R$-module, then $\mathfrak{p}=\sqrt{\operatorname{ann}_{R}(W)}$ is a prime ideal, and $\operatorname{ann}_{R}(W)$ is $\mathfrak{p}$-primary.

Definition 2.2. Let $R$ be a ring and $W$ be an $R$-module. A secondary representation of $W$ is an expression of $W$ as a sum of secondary submodules, $W=\sum_{i=1}^{t} W_{i}$, where each $W_{i}$ is called a secondary component of this representation. 
A secondary representation of $W$ is called irredundant if the prime ideals $\mathfrak{p}_{i}=\sqrt{\operatorname{ann}_{R}\left(W_{i}\right)}$ are all distinct and none of the summands $W_{i}$ can be removed from the sum. The set $\left\{\mathfrak{p}_{1}, \ldots, \mathfrak{p}_{t}\right\}$ is independent of the irredundant secondary representation and is called the set of attached primes of $W$, denoted by $\operatorname{Att}_{R}(W)$.

Clearly a secondary module has a unique attached prime. Moreover, over a local ring $(R, \mathfrak{m})$, if a nonzero module $W$ has finite length, then $W$ is secondary with $\operatorname{Att}_{R}(W)=\{\mathfrak{m}\}$. A key fact is that every Artinian $R$-module admits an irredundant secondary representation. In particular, all local cohomology modules $H_{\mathfrak{m}}^{i}(R)$ have an irredundant secondary representation.

Remark 2.3. When $(R, \mathfrak{m})$ is a complete local ring, Matlis duality induces a correspondence between (irredundant) secondary representations of Artinian modules and (irredundant) primary decompositions of Noetherian modules. In particular, if $(R, \mathfrak{m})$ is complete, and $S$ is an $n$-dimensional regular local ring mapping onto $R$, then $\operatorname{Att}_{R}\left(H_{\mathfrak{m}}^{i}(R)\right)=\operatorname{Ass}_{R}\left(\operatorname{Ext}_{S}^{n-i}(R, S)\right)$, as the Matlis dual of $H_{\mathfrak{m}}^{i}(R)$ is isomorphic to $\operatorname{Ext}_{S}^{n-i}(R, S)$.

We conclude this section by recalling the definition of surjective element and strictly filter regular element.

Definition 2.4. Let $(R, \mathfrak{m})$ be a local ring of dimension $d$. An element $x \in \mathfrak{m}$ is called a surjective element if $x \notin \mathfrak{p}$ for all $\mathfrak{p} \in \bigcup_{i=0}^{d} \operatorname{Att}_{R}\left(H_{\mathfrak{m}}^{i}(R)\right)$, and $x$ is called a strictly filter regular element if $x \notin \mathfrak{p}$ for all $\mathfrak{p} \in\left(\bigcup_{i=0}^{d} \operatorname{Att}_{R}\left(H_{\mathfrak{m}}^{i}(R)\right)\right) \backslash\{\mathfrak{m}\}$.

Remark 2.5. (1) The definition of surjective element we give is not the original one introduced by Horiuchi-Miller-Shimomoto in [HMS14]. However, note that $\operatorname{Ass}_{R}(R) \subseteq$ $\cup_{i=0}^{\operatorname{dim}(R)} \operatorname{Att}_{R}\left(H_{\mathfrak{m}}^{i}(R)\right)$ by [BS13, 11.3.9] and thus surjective elements are always nonzerodivisors. Moreover, it follows from the definition that $x$ is a surjective element if and only if $H_{\mathfrak{m}}^{i}(R) \stackrel{\cdot x}{\rightarrow} H_{\mathfrak{m}}^{i}(R)$ is surjective for each $i$. Therefore our definition is equivalent to the original definition of surjective element by [MQ18, Proposition 3.3].

(2) The definition of strictly filter regular element was originally introduced by CuongMorales-Nhan in [CMN04]. It is easy to see that $x$ is a strictly filter regular element if and only if $\operatorname{coker}\left(H_{\mathfrak{m}}^{i}(R) \stackrel{x}{\rightarrow} H_{\mathfrak{m}}^{i}(R)\right)$ has finite length for each $i$.

Surjective elements are important in the study of the deformation problem for F-injectivity. For instance, it was first proved in [HMS14, Theorem 3.7] that if $R /(x)$ is F-injective and $x$ is a surjective element, then $R$ is F-injective (see also [MQ18, Corollary 3.8] or the proof of Theorem 3.4 in the next section). In fact, we do not know any example that $R /(x)$ is F-injective but $x$ is not a surjective element, see Question 4.3.

\section{F-Stable SECONDARY REPRESEntation}

We introduce the key concept of this article.

Definition 3.1. Let $R$ be a ring of characteristic $p>0$, and let $W$ be an $R$-module with a Frobenius action $F$. We say that $W$ admits an F-stable secondary representation if there exists a secondary representation $W=\sum_{i=1}^{t} W_{i}$ such that each $W_{i}$ is F-stable, i.e., $F\left(W_{i}\right) \subseteq$ $W_{i}$ for all $i$. 
Observe that, even though we are not explicitly asking that the F-stable secondary representation is irredundant, this can always be achieved, whenever such a representation exists. It seems natural to ask when a secondary component of an Artianina module is F-stable, we show this is always the case for secondary components whose attached primes are minimal in the set of all attached primes.

Lemma 3.2. Let $R$ be a ring of characteristic $p>0$, and let $W$ be an Artinian $R$-module with a Frobenius action $F$. Let $W=\sum_{i=1}^{t} W_{i}$ be an irredundant secondary representation, with $\mathfrak{p}_{i}=\sqrt{\operatorname{ann}_{R}\left(W_{i}\right)}$. If $\mathfrak{p}_{i} \in \operatorname{MinAtt}_{R}(W)$, then $W_{i}$ is F-stable.

In particular, if $(R, \mathfrak{m})$ is a local ring of characteristic $p>0$ and dimension $d$, then $H_{\mathfrak{m}}^{d}(R)$ has an $F$-stable secondary representation.

Proof. Since $\mathfrak{p}_{i} \in \operatorname{Min} \operatorname{Att}(W)$, we can pick $y \in \cap_{j \neq i} \mathfrak{p}_{j}$ but $y \notin \mathfrak{p}_{i}$. Then $y W_{i}=W_{i}$ and $y^{N} W_{j}=0$ for all $j \neq i$ and $N \gg 0$. Therefore we have $y^{N} W=W_{i}$ for all $N \gg 0$, and thus $F\left(W_{i}\right)=F\left(y^{N} W_{i}\right) \subseteq F\left(y^{N} W\right)=y^{p N} F(W) \subseteq y^{p N} W=W_{i}$.

The last conclusion follows since it is well-known that $\operatorname{Att}_{R}\left(H_{\mathfrak{m}}^{d}(R)\right)=\{\mathfrak{p} \mid \operatorname{dim}(R / \mathfrak{p})=d\}$, see [BS13, Theorem 7.3.2], in particular, $\operatorname{Att}_{R}\left(H_{\mathfrak{m}}^{d}(R)\right)=\operatorname{MinAtt}_{R}\left(H_{\mathfrak{m}}^{d}(R)\right)$.

For secondary components whose attached primes are not necessarily minimal, the corresponding secondary components may not be F-stable. However, we do not know whether this can happen when $W$ is a local cohomology module with its natural Frobenius action, see Question 4.1.

Example 3.3. Let $R=\mathbb{F}_{p} \llbracket x, y \rrbracket$ and let $W=\mathbb{F}_{p} \oplus H_{\mathfrak{m}}^{2}(R)$. Consider the Frobenius action $F$ on $W$ that sends $(1,0)$ to $\left(1, x^{-p} y^{-1}\right)$ and is the natural one on $H_{\mathfrak{m}}^{2}(R)$. Then $F$ is injective on $W$, but we claim that $H_{\mathfrak{m}}^{2}(R)$ is the only proper nontrivial F-stable submodule of $W$. Indeed, let $0 \neq W^{\prime}$ be an F-stable submodule of $W$, it is enough to show that $0 \oplus H_{\mathfrak{m}}^{2}(R) \subseteq W^{\prime}$. Choose $a=(b, c) \neq 0$ inside $W^{\prime}$. If $c=0$, then $b \neq 0$. By replacing $a$ with $F(a)$, we can assume that $c \neq 0$. Note that $y F(a)=y F(b, 0)+(0, y F(c))=(0, y F(c)) \neq 0$ since the action $y F: H_{\mathfrak{m}}^{2}(R) \rightarrow H_{\mathfrak{m}}^{2}(R)$ is injective. Moreover $H_{\mathfrak{m}}^{2}(R)$ is simple as an $R$-module with a Frobenius action, so $0 \oplus H_{\mathfrak{m}}^{2}(R) \subseteq W^{\prime}$. Since $W$ is not secondary, this implies that there is no secondary representation of $W$ which is stable with respect to the given Frobenius action (any secondary component with attached prime $\mathfrak{m}$ is not F-stable).

We let $\mathbb{V}(x)$ denote the set of primes of $R$ which contain $x$. Our first main result is the following.

Theorem 3.4. Let $(R, \mathfrak{m})$ be a d-dimensional local ring of characteristic $p>0$ and let $x \in \mathfrak{m}$ be a nonzerodivisor on $R$. Suppose for each $i \neq d, H_{\mathfrak{m}}^{i}(R)$ admits a secondary representation in which the secondary components whose attached primes belong to $\mathbb{V}(x)$ are F-stable (e.g., $H_{\mathfrak{m}}^{i}(R)$ has an F-stable secondary representation). If $R /(x)$ is F-injective, then $x$ is a surjective element and $R$ is F-injective.

Proof. We prove by induction on $i \geqslant-1$ that multiplication by $x$ is surjective on $H_{\mathfrak{m}}^{i}(R)$ and that $x^{p^{e}-1} F^{e}$ is injective on $H_{\mathfrak{m}}^{i}(R)$ for all $e>0$. This will conclude the proof, since the first assertion implies $x$ is a surjective element and the second assertion implies $F$ is injective on $H_{\mathfrak{m}}^{i}(R)$ for all $i$. The base case $i=-1$ is trivial. Suppose both assertions hold for $i-1$; we 
show them for $i$. Consider the following commutative diagram:

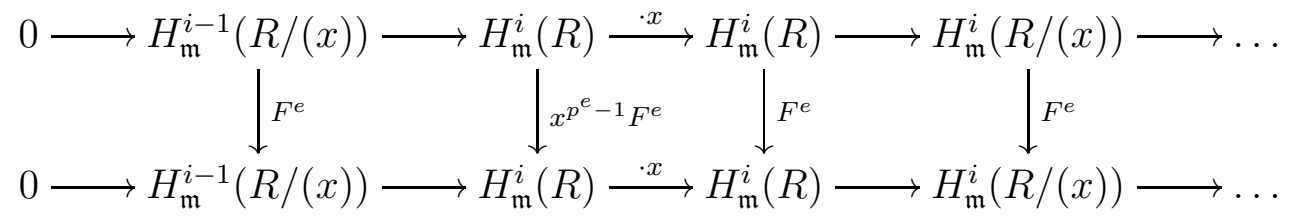

where injectivity on the left of the rows follows from our inductive hypotheses. Let $u \in$ $\operatorname{soc}\left(H_{\mathfrak{m}}^{i}(R)\right) \cap \operatorname{ker}\left(x^{p^{e}-1} F^{e}\right)$. Then $x u=0$, and thus $u$ is the image of an element $v \in$ $H_{\mathfrak{m}}^{i-1}(R /(x))$. Chasing the diagram shows that $F^{e}(v)=0$. But since $R /(x)$ is F-injective, $F^{e}$ is injective on $H_{\mathfrak{m}}^{i-1}(R /(x))$ for all $e>0$, so $v=0$ and thus $u=0$. This shows that $x^{p^{e}-1} F^{e}$ is injective on $H_{\mathfrak{m}}^{i}(R)$ for all $e>0$.

It remains to show that multiplication by $x$ is surjective on $H_{\mathfrak{m}}^{i}(R)$. Let $H_{\mathfrak{m}}^{i}(R)=\sum W_{j}$ be the secondary representation that satisfies the conditions of the theorem (note that $H_{\mathfrak{m}}^{d}(R)$ always has an F-stable secondary representation by Lemma 3.2). If there exists $W_{j} \neq 0$ whose attached prime $\mathfrak{p}_{j} \in \mathbb{V}(x)$, then it follows from the assumptions that $W_{j}$ is F-stable. Thus $x^{p^{e}-1} F^{e}\left(W_{j}\right) \subseteq x^{p^{e}-1} W_{j}=0$ for all $e \gg 0$ (since $\left.x \in \mathfrak{p}_{j}=\sqrt{\operatorname{ann}_{R}\left(W_{j}\right)}\right)$. However, we have proved that $x^{p^{e}-1} F^{e}$ is injective on $H_{\mathfrak{m}}^{i}(R)$ for all $e>0$, this implies $W_{j}=0$ and we arrive at a contradiction. Therefore $x \notin \mathfrak{p}$ for all $\mathfrak{p} \in \operatorname{Att}_{R}\left(H_{\mathfrak{m}}^{i}(R)\right)$, i.e., multiplication by $x$ is surjective on $H_{\mathfrak{m}}^{i}(R)$.

Corollary 3.5. Let $(R, \mathfrak{m})$ be a d-dimensional local ring of characteristic $p>0$ and let $x \in \mathfrak{m}$ be a nonzerodivisor on $R$. Suppose that $\operatorname{Att}_{R}\left(H_{\mathfrak{m}}^{i}(R)\right) \cap \mathbb{V}(x) \subseteq \operatorname{MinAtt}_{R}\left(H_{\mathfrak{m}}^{i}(R)\right)$ for all $i \neq d$ (e.g., when each $H_{\mathfrak{m}}^{i}(R)$ has no embedded attached primes). If $R /(x)$ is $F$-injective, then $x$ is a surjective element and $R$ is F-injective.

Proof. By Lemma 3.2, every irredundant secondary representation of $H_{\mathfrak{m}}^{i}(R)$ satisfies the assumptions of Theorem 3.4 so the conclusion follows.

We next exhibit an explicit new class of rings for which deformation of F-injectivity holds. Recall that a finitely generated $R$-module $M$ is called sequentially Cohen-Macaulay if there exists a finite filtration $0=M_{0} \subseteq M_{1} \subseteq M_{2} \subseteq \cdots \subseteq M_{n}=M$ such that each $M_{i+1} / M_{i}$ is Cohen-Macaulay and $\operatorname{dim}\left(M_{i} / M_{i-1}\right)<\operatorname{dim}\left(M_{i+1} / M_{i}\right)$. A local ring $(R, \mathfrak{m})$ is called sequentially Cohen-Macaulay if $R$ is sequentially Cohen-Macaulay as an $R$-module.

Corollary 3.6. Let $(R, \mathfrak{m})$ be a d-dimensional sequentially Cohen-Macaulay local ring of characteristic $p>0$ and let $x \in \mathfrak{m}$ be a nonzerodivisor on $R$. If $R /(x)$ is F-injective, then $x$ is a surjective element and $R$ is F-injective.

Proof. First we observe that $R$ is sequentially Cohen-Macaulay implies $\widehat{R}$ is sequentially Cohen-Macaulay and whether $R$ is F-injective (and whether $x$ is a surjective element) is unaffected by passing to the completion. Therefore we may assume $R$ is complete and thus $R$ is a homomorphic image of a regular local ring $S$. By [HS02, Theorem 1.4], $R$ is sequentially Cohen-Macaulay is equivalent to saying that, for each $0 \leqslant i \leqslant d, \operatorname{Ext}_{S}^{\operatorname{dim}(S)-i}(R, S)$ is either zero or Cohen-Macaulay of dimension $i$. In particular, $\operatorname{Ext}_{S}^{\operatorname{dim}(S)-i}(R, S)$ has no embedded associated primes and hence by Remark 2.3, $H_{\mathfrak{m}}^{i}(R)$ has no embedded attached primes for each $0 \leqslant i \leqslant d$, that is, $\operatorname{Att}_{R}\left(H_{\mathfrak{m}}^{i}(R)\right)=\operatorname{Min} \operatorname{Att}_{R}\left(H_{\mathfrak{m}}^{i}(R)\right)$. The conclusion now follows from Corollary 3.5. 
3.1. Results on local rings with perfect residue field. If we assume the residue field of $(R, \mathfrak{m})$ is perfect, then we can prove some slight stronger results. The arguments are based on appropriate modifications of the proof of Theorem 3.4, together with some ideas employed in [MQ18, Section 5]. First, we make a modification of the definition of F-stable secondary representation.

Definition 3.7. Let $R$ be a ring of characteristic $p>0$ and $\mathfrak{m}$ be a maximal ideal of $R$. Let $W$ be an $R$-module with a Frobenius action $F$. We say that $W$ admits an $\mathrm{F}^{\circ}$-stable secondary representation if there exists a secondary representation $W=\sum_{i=1}^{t} W_{i}$ such that $W_{i}$ is F-stable for all $i$ such that $\operatorname{Att}_{R}\left(W_{i}\right) \neq\{\mathfrak{m}\}$.

Theorem 3.8. Let $(R, \mathfrak{m})$ be a d-dimensional local ring of characteristic $p>0$ with perfect residue field, and let $x \in \mathfrak{m}$ be a nonzerodivisor on $R$. Suppose for each $i \neq d, H_{\mathfrak{m}}^{i}(R) \neq 0$ admits a secondary representation in which the secondary components whose attached primes belong to $\mathbb{V}(x) \backslash\{\mathfrak{m}\}$ are $F$-stable (e.g., $H_{\mathfrak{m}}^{i}(R)$ has an $\mathrm{F}^{\circ}$-stable secondary representation). If $R /(x)$ is F-injective, then $x$ is a strictly filter regular element and $R$ is F-injective.

Proof. For every $i$, we let $L_{i}=\operatorname{coker}\left(H_{\mathfrak{m}}^{i}(R) \stackrel{x}{\rightarrow} H_{\mathfrak{m}}^{i}(R)\right)$. We prove by induction on $i \geqslant-1$ that $L_{i}$ has finite length and that the Frobenius action $x^{p^{e}-1} F^{e}$ on $H_{\mathfrak{m}}^{i}(R)$ is injective for all $e>0$. This will conclude the proof, since the first assertion implies $x$ is a strictly filter regular element and the second assertion implies $F$ is injective on $H_{\mathfrak{m}}^{i}(R)$ for all $i$. The initial case $i=-1$ is trivial. Suppose both assertions hold for $i-1$; we show them for $i$. Consider the following commutative diagram:

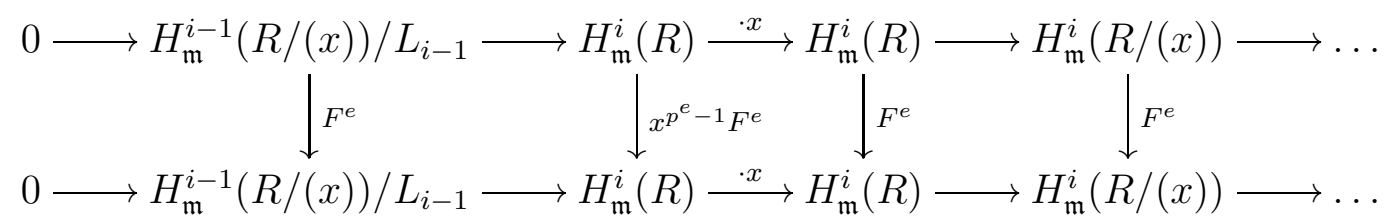

Since $L_{i-1}$ has finite length, $F^{e}$ is injective on $H_{\mathfrak{m}}^{i-1}(R /(x))$ by assumption, and $R / \mathfrak{m}$ is perfect, we have that $F^{e}$ induces a bijection on $L_{i-1} \subseteq H_{\mathfrak{m}}^{i-1}(R /(x))$. Thus, $F^{e}$ induces an injection on $H_{\mathfrak{m}}^{i-1}(R /(x)) / L_{i-1}$ for all $e>0$. Therefore, chasing the diagram above as in the proof of Theorem 3.4 we know that $x^{p^{e}-1} F^{e}$ is injective on $H_{\mathfrak{m}}^{i}(R)$ for all $e>0$.

It remains to show that $L_{i}$ has finite length. If there exists $W_{j} \neq 0$ whose attached prime $\mathfrak{p}_{j} \in \mathbb{V}(x) \backslash\{\mathfrak{m}\}$, then it follows from the assumptions that $W$ is F-stable (note that $H_{\mathfrak{m}}^{d}(R)$ always has an F-stable secondary representation by Lemma 3.2). Thus $x^{p^{e}-1} F^{e}\left(W_{j}\right) \subseteq$ $x^{p^{e}-1} W_{j}=0$ for all $e \gg 0$ (since $x \in \mathfrak{p}_{j}=\sqrt{\operatorname{ann}_{R}\left(W_{j}\right)}$ ). However, we have proved that $x^{p^{e}-1} F^{e}$ is injective on $H_{\mathfrak{m}}^{i}(R)$ for all $e>0$, this implies $W_{j}=0$ and we arrive at a contradiction. Therefore $x \notin \mathfrak{p}$ for all $\mathfrak{p} \in \operatorname{Att}_{R}\left(H_{\mathfrak{m}}^{i}(R)\right) \backslash\{\mathfrak{m}\}$, i.e., $L_{i}=\operatorname{coker}\left(H_{\mathfrak{m}}^{i}(R) \stackrel{x}{\rightarrow}\right.$ $\left.H_{\mathfrak{m}}^{i}(R)\right)$ has finite length.

Corollary 3.9. Let $(R, \mathfrak{m})$ be a d-dimensional local ring of characteristic $p>0$ with perfect residue field, and let $x \in \mathfrak{m}$ be a nonzerodivisor on $R$. Suppose that $\operatorname{Att}_{R}\left(H_{\mathfrak{m}}^{i}(R)\right) \cap \mathbb{V}(x) \subseteq$ $\operatorname{MinAtt}_{R}\left(H_{\mathfrak{m}}^{i}(R)\right) \cup\{\mathfrak{m}\}$ for all $i \neq d$. If $R /(x)$ is F-injective, then $x$ is a strictly filter regular element and $R$ is F-injective. In particular, F-injectivity deforms if $\operatorname{dim}\left(R / \operatorname{ann}_{R}\left(H_{\mathfrak{m}}^{i}(R)\right)\right) \leqslant$ 1 for all $i \neq d$ and $R / \mathfrak{m}$ is perfect.

Proof. By Lemma 3.2, every irredundant secondary representation of $H_{\mathfrak{m}}^{i}(R)$ satisfies the assumptions of Theorem 3.8 so the first conclusion follows. To see the second conclusion, 
it is enough to observe that when $\operatorname{dim}\left(R / \operatorname{ann}_{R}\left(H_{\mathfrak{m}}^{i}(R)\right)\right) \leqslant 1$, we have $\operatorname{Att}_{R}\left(H_{\mathfrak{m}}^{i}(R)\right) \subseteq$ $\operatorname{MinAtt}_{R}\left(H_{\mathfrak{m}}^{i}(R)\right) \cup\{\mathfrak{m}\}$.

3.2. Results on $\mathbb{N}$-graded rings. For the rest of this section, we assume that $(R, \mathfrak{m}, k)$ is an $\mathbb{N}$-graded algebra over a field $k$ of characteristic $p>0$ ( $k$ is not necessarily perfect). Given a graded module $W=\bigoplus_{j} W_{j}$ and $a \in \mathbb{Z}$, we denote by $W(a)$ the shift of $W$ by $a$, that is, the graded $R$-module such that $W(a)_{j}=W_{a+j}$. In this context, when talking about a Frobenius action $F$ on a graded module $W$, we insist that $\operatorname{deg}(F(\eta))=p \cdot \operatorname{deg}(\eta)$ for all homogeneous $\eta \in W$. This is the case for the natural Frobenius action $F$ on the local cohomology modules $H_{\mathfrak{m}}^{i}(R)$.

The goal of this subsection is to extend Theorem 3.8 in this $\mathbb{N}$-graded setting, by removing the assumption that the residue field $k$ is perfect and by strengthening the conclusion to that $x$ is actually a surjective element.

Theorem 3.10. Let $(R, \mathfrak{m}, k)$ be a d-dimensional $\mathbb{N}$-graded $k$-algebra of characteristic $p>0$ and let $x \in \mathfrak{m}$ be a homogeneous nonzerodivisor on $R$. Suppose for each $i \neq d, H_{\mathfrak{m}}^{i}(R)$ admits a secondary representation in which the secondary components whose attached primes belong to $\mathbb{V}(x) \backslash\{\mathfrak{m}\}$ are F-stable (e.g., $H_{\mathfrak{m}}^{i}(R)$ has an $\mathrm{F}^{\circ}$-stable secondary representation). If $R /(x)$ is F-injective, then $x$ is a surjective element and $R$ is $F$-injective.

Proof. Let $\operatorname{deg}(x)=t>0$. We have a graded long exact sequence of local cohomology, induced by the short exact sequence $0 \rightarrow R(-t) \stackrel{x}{\rightarrow} R \rightarrow R /(x) \rightarrow 0$. Moreover, this exact sequence fits in the commutative diagram:

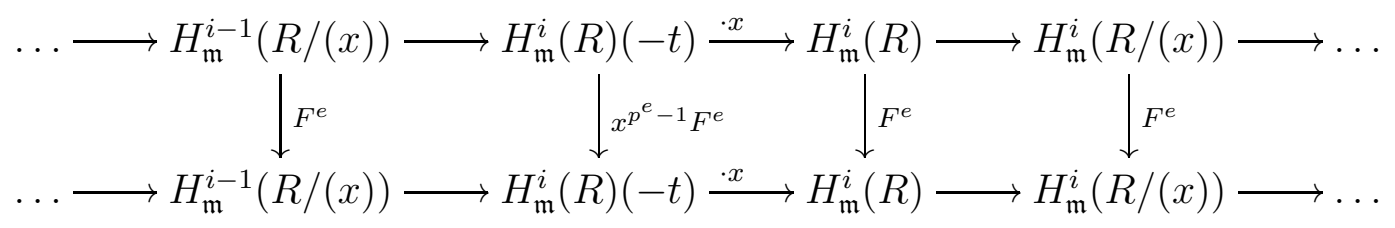

Observe that all the Frobenius actions are compatible with the grading. We show by induction on $i \geqslant-1$ that the map $H_{\mathfrak{m}}^{i}(R)(-t) \stackrel{x}{\rightarrow} H_{\mathfrak{m}}^{i}(R)$ is surjective and that $x^{p^{e}-1} F^{e}$ is injective on $H_{\mathfrak{m}}^{i}(R)(-t)$. This will conclude the proof, since the first assertion implies $x$ is a surjective element and the second assertion implies $F$ is injective on $H_{\mathfrak{m}}^{i}(R)$ for all $i$. The base case $i=-1$ is trivial. Suppose both assertions hold for $i-1$; we show them for $i$. By the same argument as in the proof of Theorem 3.4, we have that $x^{p^{e}-1} F^{e}$ is injective on $H_{\mathfrak{m}}^{i}(R)(-t)$ for all $e>0$.

It remains to show that multiplication by $x$ map $H_{\mathfrak{m}}^{i}(R)(-t) \stackrel{x}{\rightarrow} H_{\mathfrak{m}}^{i}(R)$ is surjective. Now by the same argument as in the proof of Theorem 3.8, we know that $L_{i}=\operatorname{coker}\left(H_{\mathfrak{m}}^{i}(R)(-t) \stackrel{x}{\rightarrow}\right.$ $H_{\mathfrak{m}}^{i}(R)$ ) has finite length (note that we can ignore the graded structure here). Finally, consider the following commutative diagram:

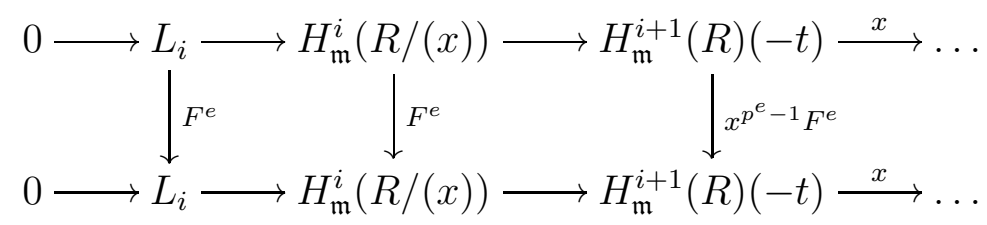

Since $F^{e}$ is injective on $H_{\mathfrak{m}}^{i}(R /(x))$ by assumption, it is also injective on $L_{i}$. But since the finite length module $L_{i}$ is graded and the Frobenius action is compatible with the grading 
(as the action is induced from $H_{\mathfrak{m}}^{i}(R /(x))$ ), this forces $L_{i}$ to be concentrated in degree zero. If $L_{i} \neq 0$, then $\left[L_{i}\right]_{0} \cong\left[H_{\mathfrak{m}}^{i}(R) / x H_{\mathfrak{m}}^{i}(R)(-t)\right]_{0} \neq 0$, in particular $\left[H_{\mathfrak{m}}^{i}(R)\right]_{0} \neq 0$. However, this implies the existence of a nonzero element $u \in\left[H_{\mathfrak{m}}^{i}(R)(-t)\right]_{t}$. Since we have proved that $x^{p^{e}-1} F^{e}$ is injective on $H_{\mathfrak{m}}^{i}(R)(-t)$, this gives a nonzero element $x^{p^{e}-1} F^{e}(u)$ in degree $p^{e} t>0$ for all $e>0$, which is a contradiction because $\left[H_{\mathfrak{m}}^{i}(R)(-t)\right]_{\gg 0}=0$ (here we are using that the Frobenius action $x^{p^{e}-1} F^{e}$ is compatible with the grading on $H_{\mathfrak{m}}^{i}(R)(-t)$, that is, $\operatorname{deg}\left(x^{p^{e}-1} F^{e}(\eta)\right)=p^{e} \operatorname{deg}(\eta)$ for all $\left.\eta \in H_{\mathfrak{m}}^{i}(R)(-t)\right)$. Therefore $L_{i}=0$, i.e., the multiplication by $x$ map $H_{\mathfrak{m}}^{i}(R)(-t) \stackrel{x}{\rightarrow} H_{\mathfrak{m}}^{i}(R)$ is surjective.

Corollary 3.11. Let $(R, \mathfrak{m}, k)$ be a d-dimensional $\mathbb{N}$-graded $k$-algebra of characteristic $p>0$ and let $x \in \mathfrak{m}$ be a homogeneous nonzerodivisor on $R$. Suppose that $\operatorname{Att}_{R}\left(H_{\mathfrak{m}}^{i}(R)\right) \cap \mathbb{V}(x) \subseteq$ $\operatorname{MinAtt}_{R}\left(H_{\mathfrak{m}}^{i}(R)\right) \cup\{\mathfrak{m}\}$ for all $i \neq d$ (e.g., $x$ is a strictly filter regular element). If $R /(x)$ is $F$-injective, then $x$ is a surjective element and $R$ is F-injective.

Proof. By Lemma 3.2, every irredundant secondary representation of $H_{\mathfrak{m}}^{i}(R)$ satisfies the assumptions of Theorem 3.10 so the conclusion follows.

\section{Ending QUESTIONS AND REMARKS}

We end by collecting some questions that arise from the results in this article. Motivated by Definition 3.1 and Theorem 3.4, it is natural to ask the following.

Question 4.1. Let $(R, \mathfrak{m})$ be a local ring of characteristic $p>0$. If $H_{\mathfrak{m}}^{i}(R) \neq 0$, does it admit an F-stable secondary representation?

By Theorem 3.4, a positive answer to Question 4.1 implies that F-injectivity deforms.

Question 4.2. Let $(R, \mathfrak{m})$ be a local ring of characteristic $p>0$. If $H_{\mathfrak{m}}^{i}(R) \neq 0$, does it admit a secondary representation such that the secondary component with attached prime $\mathfrak{m}$, if not zero, is F-stable?

This is weaker than Question 4.1, but an affirmative answer also implies that F-injectivity deforms. Suppose $R /(x)$ is F-injective, we will show $x$ is a surjective element and thus $R$ is F-injective by [HMS14, Theorem 3.7] (or use the same argument as in Theorem 3.4). In fact, if $x \in \mathfrak{p}$ for some $\mathfrak{p} \in \operatorname{Att}_{R}\left(H_{\mathfrak{m}}^{i}(R)\right)$, then $x \in \mathfrak{p} R_{\mathfrak{p}} \in \operatorname{Att}_{R_{\mathfrak{p}}}\left(H_{\mathfrak{p} R_{\mathfrak{p}}}^{j}\left(R_{\mathfrak{p}}\right)\right)$ for some $j$ and $R_{\mathfrak{p}} / x R_{\mathfrak{p}}$ is still F-injective. Now an affirmative answer to Question 4.2 applied to $\left(R_{\mathfrak{p}}, \mathfrak{p} R_{\mathfrak{p}}\right)$ implies that there exists a nonzero secondary component of $H_{\mathfrak{p} R_{\mathfrak{p}}}^{j}\left(R_{\mathfrak{p}}\right)$ with attached prime $\mathfrak{p} R_{\mathfrak{p}}$ that is F-stable, and we can argue as in the proof of Theorem 3.4 to arrive at a contradiction.

Question 4.3. Let $(R, \mathfrak{m})$ be a local ring of characteristic $p>0$, and let $x \in \mathfrak{m}$ be a nonzerodivisor on $R$. If $R /(x)$ is F-injective, is it true that $\mathfrak{m} \notin \operatorname{Att}\left(H_{\mathfrak{m}}^{i}(R)\right)$ for all $i$ ?

Similar to the discussion above, we point out that an affirmative answer to Question 4.3 also implies that $x$ is a surjective element (and hence implies that F-injectivity deforms): if not, then $x \in \mathfrak{p}$ for some $\mathfrak{p} \in \operatorname{Att}_{R}\left(H_{\mathfrak{m}}^{i}(R)\right)$, but then $R_{\mathfrak{p}} / x R_{\mathfrak{p}}$ is still F-injective and $\mathfrak{p} R_{\mathfrak{p}} \in \operatorname{Att}_{R_{\mathfrak{p}}}\left(H_{\mathfrak{p} R_{\mathfrak{p}}}^{j}\left(R_{\mathfrak{p}}\right)\right)$ for some $j$, which contradicts Question 4.3 for $\left(R_{\mathfrak{p}}, \mathfrak{p} R_{\mathfrak{p}}\right)$.

\section{REFERENCES}

[BS13] M. P. Brodmann and R. Y. Sharp. Local cohomology, volume 136 of Cambridge Studies in Advanced Mathematics. Cambridge University Press, Cambridge, second edition, 2013. An algebraic introduction with geometric applications. 2, 3, 4 
[CMN04] Nguyen Tu Cuong, Marcel Morales, and Le Thanh Nhan. The finiteness of certain sets of attached prime ideals and the length of generalized fractions. J. Pure Appl. Algebra, 189(1-3):109-121, 2004. 3

[Fed83] Richard Fedder. F-purity and rational singularity. Trans. Amer. Math. Soc., 278(2):461-480, 1983. 1

[HH94] Melvin Hochster and Craig Huneke. F-regularity, test elements, and smooth base change. Trans. Amer. Math. Soc., 346(1):1-62, 1994. 1

[HMS14] Jun Horiuchi, Lance Edward Miller, and Kazuma Shimomoto. Deformation of $F$-injectivity and local cohomology. Indiana Univ. Math. J., 63(4):1139-1157, 2014. With an appendix by Karl Schwede and Anurag K. Singh. 1, 3, 8

[HS02] Jürgen Herzog and Enrico Sbarra. Sequentially Cohen-Macaulay modules and local cohomology. In Algebra, arithmetic and geometry, Part I, II (Mumbai, 2000), volume 16 of Tata Inst. Fund. Res. Stud. Math., pages 327-340. Tata Inst. Fund. Res., Bombay, 2002. 5

[MQ18] Linquan Ma and Pham Hung Quy. Frobenius actions on local cohomology modules and deformation. Nagoya Math. J., 232:55-75, 2018. 1, 3, 6

[Sin99a] Anurag K. Singh. Deformation of F-purity and F-regularity. J. Pure Appl. Algebra, 140(2):137148, 1999. 1

[Sin99b] Anurag K. Singh. F-regularity does not deform. Amer. J. Math., 121(4):919-929, 1999. 1

Dipartimento di Matematica, Università di Genova, Via Dodecaneso 35, 16146 Genova, ITALY

Email address: destefani@dima.unige.it

Department of Mathematics, Purdue University, West Lafayette, IN 47907, USA

Email address: ma326@purdue.edu 